\title{
Inhaltsverzeichnis
}

Grußwort

Geleitwort IX

Verzeichnis der wissenschaftlichen Veröffentlichungen XXI

Autorenverzeichnis XXVII

MARTIN AHRENS

Verewigte Insolvenzverfahren reloaded

- ein Problem asymmetrischer Verfahren 1

CHRISTIAN BERGER

Wegfall des Anfechtungsanspruchs bei „Massezulänglichkeit“

GEORG BITTER

Die Doppelsicherung durch Gesellschaft und Gesellschafter als

Lackmustest für den Normzweck des Gesellschafterdar-

lehensrechts

REINHARD BORK

Heilung der Anfechtbarkeit?

MAX BRAEUER

Rechtsanwälte vor dem Bundesgerichtshof 77

MORITZ BRINKMANN

Der Insolvenzexperte in der Eigenverwaltung

- Notwendigkeit, Bestellung, Haftung 99

PETER DePRÉ

Rechtsetzung oder noch Rechtsfortbildung

- Reflexion über den Beschluss des Bundesgerichtshofs vom

14. Juli 2016 - IX ZB 31/14 -

SUSANNE DORNBLÜTH

Mediation als Haftungsfalle?

- Besondere Pflichten von juristisch ausgebildeten Mediatoren - 
INGO DRESCHER

Die Geschäftsführerhaftung nach $\mathbb{} \$ 64$ Satz $1 \mathrm{GmbHG}$ und die Insolvenzanfechtung

JÜRGEN ELLENBERGER

Lastschrift in der Insolvenz

DETLEV FISCHER

Maklerrechtliche Spuren und Impulse in der Judikatur des

IX. Zivilsenats des Bundesgerichtshofs

GERO FISCHER

Können Massegläubiger eine Umqualifizierung ihrer Forderung

in eine Insolvenzforderung erreichen?

LUCAS F. FLÖTHER

Die Ziele des präventiven Restrukturierungsrahmens

- Kontinuität und Wandel -

HANS GERHARD GANTER

Das Vermieterpfandrecht an volatilen Gegenständen in der

Mieterinsolvenz

MARKUS GEHRLEIN

Vorvertrag und Option in der Insolvenz

ARNDT GEIWITZ

Die Besteuerung im Rahmen von Insolvenz und Restrukturierung

- Anspruch und Wirklichkeit -

THORSTEN GRAEBER

Vergütung von Insolvenzverwaltern - eine Sonderrechtszone? ..... 287

DIETMAR GRUPP

Präklusion im Wandel der Zeit

ULRICH HAAS

Umsetzung des präventiven Restrukturierungsrahmens an der

Schnittstelle zwischen Insolvenz- und Gesellschaftsrecht

PETRA HEIDENFELDER

Was hat sich an der Rechtspersönlichkeit, der die Insolvenz droht, im Verlauf der gesetzlichen Entwicklung verändert? 


\section{HERIBERT HIRTE}

Die Reform des Insolvenzanfechtungsrechts in der politischen

Diskussion

JOST HÜTTENBRINK

Die Verschwiegenheitspflicht der Vorstandsmitglieder einer

Rechtsanwaltskammer im Spannungsfeld zur Informations-

freiheit

FLORIAN JACOBY

Masseverbindlichkeiten und anfechtungsfeste Deckungen aus dem

Eröffnungsverfahren

GÜNTER KAHLERT

Der Ertragsteueranspruch als Insolvenzforderung im Wandel der

Rechtsprechung des Bundesfinanzhofes 405

FRANK KEBEKUS UND DAVID GEORG

Eigenständiger Vergütungsantrag des „nur“ vorläufigen

Sachwalters

DETLEF KLEINDIEK

Geschäftsführung und Ressortaufteilung in der Unternehmens-

krise

BRUNO M. KÜBLER UND DIETMAR RENDELS

Insolvenzplan: Kein Obstruktionsverbot bei Schuldner-/

Gesellschaftervorteilen? 465

KAREN KUDER UND KATRIN STOHRER

Aktuelles zur Rechtshandlung des Schuldners nach $\$ 133$

Abs. 1 InsO 481

PeTER LaRoche

Die Insolvenz natürlicher Personen als Herausforderung

des Internationalen Insolvenzrechts

ILSE LOHMANN

Die Festschrift - Risiken und Nebenwirkungen

STEPHAN MADAUS

Roma locuta, causa finita?

- Folgefragen aus dem Urteil des Bundesgerichtshofes zur

Haftung der Geschäftsleiter in der Eigenverwaltung - 
WOLFGANG MAROTZKE

Fluch der bösen Tat

- Zum richtigen Umgang mit $\mathbb{} 14$ Abs. $3 \mathrm{InsO}-$

THOMAS MEHRING

Objektive Gläubigerbenachteiligung und Saldierung mit Vorteilen der Masse

PRAXEDIS MÖHRING

Fortführung des Geschäftsbetriebs im Eröffnungsverfahren

durch den schwachen vorläufigen Insolvenzverwalter?

CHRISTOPH NIERING UND DANIEL BERGNER

„Insolvenzverfahren 4.0“

- Gestaltungselemente einer Modernisierung des Insolvenz-

verfahrens -

DIETMAR ONUSSEIT

Quotenzahlung und Vorsteuerberichtigung

KLAUS PANNEN

Die Bank als Beteiligte im vorinsolvenzlichen

Restrukturierungsverfahren des Schuldners

- Chancen und Risiken für Kreditinstitute im vorinsolvenzlichen

Restrukturierungsverfahren -

GERHARD PAPE

Hoffnungsschimmer Restrukturierungsrichtlinie

- neue Impulse für die Entschuldung aus Europa -

ANDREAS PIEKENBROCK

Zahlungen aus debitorischen Konten im Insolvenzan-

fechtungsrecht

Michael Pluta UND GRIT HeIDRICH

Die Rückforderung festgesetzter, im Nachhinein als verwirkt

erkannter Verwaltervergütungen

HANNS PRÜTTING

Die Ablehnung eines Richters wegen Besorgnis der Befangenheit

BERND RAEBEL

Gemeinnützigkeit, Vereinigungsfreiheit und Gleichheitssätze 


\section{ANDREAS REMMERT}

Anwaltliches Berufsrecht und Insolvenzrecht:

Verbindungslinien

ALEXANDER RIEDEL

Schenkungsanfechtung gemäß $\mathbb{1 3 4} \mathrm{Ins} \mathrm{O}$ 787

SUSANNE RIEDEMANN

Die Kontenführung im Insolvenzverfahren 799

\section{STEPHAN RIES}

Der Bundesfinanzhof und die Bindung seiner Richter an Recht und Gesetz (Art. 20 Abs. 3 GG)

- auch gegenüber der spezialgesetzlichen „Rangordnung“

bei Insolvenz -

INGO SAENGER

Der unparteiliche Richter in der juristischen Fachwelt

CARSTEN SCHÄFER

Einbeziehung der Gesellschafter in ein vorinsolvenzliches

Restrukturierungsverfahren?

- Empfehlungen zur Umsetzung der

EU-Restrukturierungsrichtlinie -

JENS M. SCHMITTMANN

Insolvenzverwalter und Berufsgerichtsbarkeit

HEINRICH SCHOPPMEYER

Schenkungsanfechtung bei Restschuldversicherungen

VOLKER SCHULTZ

Der Restschuldbefreiungstourismus im Lichte der neuen

EuInsVO

WERNER STERNAL

Anfechtbarkeit von Honorarzahlungen an Sanierungsberater

im Lichte der Rechtsprechung des Bundesgerichtshofes

CHRISTOPH THOLE

Auskunftsansprüche des Insolvenzverwalters gegen (mögliche)

Anfechtungsgegner in der höchstrichterlichen Rechtsprechung .... 963 


\section{SVEN-HOLGER UNDRITZ}

Die Patronatserklärung als unentgeltliche Leistung in der Insolvenz des Patrons ( $\mathbb{S} 39$ Abs. 1 Nr. 4, 134 InsO)

\section{HEINZ VALLENDER}

Restrukturierung im Land der Pharaonen

- Das neue ägyptische Insolvenzrecht auf dem Prüfstand GERHARD VILL

Die Vergütung von Verwalter oder Sachwalter in Insolvenzverfahren mit hoher Gläubigerzahl

ERICH WACLAWIK

Das Mitverschulden des Mandanten bei beschränktem Mandat des steuerlichen Beraters

ALEXANDER WEINLAND

Insolvenzrechtliche Betrachtungen zur neuen

Musterfeststellungsklage

LARS WESTPFAHL

Der Überschuldungstatbestand aus der Sicht eines Praktikers

- Plädoyer für die Abschaffung der Überschuldung als

Insolvenzauslöser -

THOMAS WINTER

Drittschutzwille und Vertrauen bei Beratungsverträgen

HELMUT ZiPPERER

Auf Erkundung zu gemeinsamen europäischen Rechtsüberzeugungen 